\title{
ESTILOS DE VIDA DE CAMPESINOS Y PESCADORES OCASIONALES RESIDENTES EN YAGUAJAY, SANCTI SPÍRITUS, CUBA
}

\section{LIFESTYLES OF FARMERS AND OCCASIONALLY FISHERMEN RESIDENTS IN YAGUAJAY, SANCTI SPÍRITUS, CUBA}

\author{
Vanessa Vázquez Sánchez ${ }^{*}$, Armando Rangel Rivero1, Sairys Peña Alcolea1, Yoandry Alfonso \\ Díaz Fuentes², Victoria Constanza Ramenzoni³ , Dairon Antonio Ojeda Martínez ${ }^{4}$
}

\author{
${ }^{I}$ Museo Antropológico Montané. Facultad de Biología. Universidad de La Habana. Cuba \\ ${ }^{2}$ Instituto Nacional de Higiene, Epidemiología y Microbiología. La Habana, Cuba \\ ${ }^{3}$ Departamento de Ecología Humana. Escuela de Ciencias Biológicas y ambientales. Universidad de Rutgers, New Brunswick. New \\ Jersey. Estados Unidos \\ ${ }^{4}$ Universidad de Salford. Reino Unido
}

PALABRAS CLAVE: pescadores; campesinos; dieta; estado nutricional; actividad física

RESUMEN La pesca y la agricultura requieren de un gasto de energía elevado, lo cual influye en los estilos de vida de las poblaciones que practican estas actividades. El objetivo del trabajo es comparar composición de la dieta, estado nutricional y actividad física de 19 campesinos y 14 pescadores ocasionales del municipio Yaguajay, Cuba. La frecuencia de consumo semanal de alimentos se obtuvo mediante un cuestionario. Se evaluó el estado nutricional y la actividad física con el cuestionario IPAQ, este último se aplicó a una submuestra, a la cual se le monitoreó su estado nutricional cinco meses después de la evaluación inicial. La dieta de ambos grupos se caracterizó por alto consumo de arroz, frijoles y viandas. El consumo de pescados y vegetales fue mayor en los pescadores. En la segunda medición, los pescadores aumentaron aproximadamente una unidad su porcentaje de grasa corporal, mientras que los campesinos perdieron aproximadamente $1 \mathrm{~kg}$ de peso. El $60 \%$ de los pescadores realizó actividad física alta y el $40 \%$ media, para los campesinos los valores fueron $88,9 \%$ y $11,1 \%$ respectivamente. Los campesinos practicaban actividad física diaria, mientras que los pescadores pescaban ocasionalmente. A su vez, los pescadores tuvieron una dieta más saludable, aunque fue más frecuente en ellos la obesidad. Rev Arg Antrop Biol 22(2), 2020. doi: 10.24215/18536387e017

\section{KEYWORDS fishermen; farmers; diet; nutritional state; physical activity}

$A B S T R A C T$ Fishing and agriculture require a high energy expenditure that influences lifestyles of populations that practice these activities. The goal of the paper is to compare eating habits, nutritional status and physical activity of 19 farmers and 14 occasionally fishermen of the municipality Yaguajay, Cuba. Questionnaires of frequency of weekly foods consumption were applied. The nutritional status was evaluated. The physical activity was determined using questionnaire IPAQ short version in a subsample that were measured again five months later in order to evaluate nutritional status. In both groups diet was characterized by high consumption of rice, beans and "viandas". The fishermen had more consumption of fish and vegetables. In the second mensuration, fishermen increased in approximately one unit their percentage of body fat, while farmers lost $1 \mathrm{~kg}$ of weight approximately. $60 \%$ of fishermen were classified with a category of high physical activity and $40 \%$ with medium, and for farmers the frequencies are of $88,9 \%$ and $11,1 \%$ respectively. It's concluded that farmers do physical activity daily; while the selected fishermen fish occasionally also they had a healthier diet but they are more obese than farmers. Rev Arg Antrop Biol 22(2), 2020. doi: 10.24215/18536387e017
El término estilo de vida puede ser definido de diversas maneras. Es la elección de un conjunto de actividades relacionadas con distintas esferas como la producción, la actividad social, vida cotidiana, tiempo libre y el sistema de hábitos: alimentarios, de cultura física, sueño, entre otros. Está basado en patrones de comportamiento que estructuran la organización temporal, el sistema de relaciones sociales y las pautas de consumo de un grupo de individuos (Álvarez, 2009).
Numerosas variables se emplean en el estudio de los estilos de vida de las poblaciones hu-

Financiamiento: Proyecto UH (Universidad de La Habana) 04.25.88.18. *Correspondencia a: Vanessa Vázquez Sánchez. Email de contacto: vanevaz@fbio.uh.cu

Recibido 21 Noviembre 2018; aceptado 5 Agosto 2019

doi: $10.24215 / 18536387 \mathrm{e} 017$ 
manas, entre ellas la dieta, el estado nutricional y la actividad física. Los estilos de vida de las personas a su vez, están condicionados por factores biológicos, ecológicos y culturales. Entre estos últimos destaca la ocupación laboral. Algunos trabajos como la agricultura y la pesca se caracterizan por un alto gasto energético e implican interacción con los ecosistemas naturales. Por lo tanto, se infiere que las características del medio, así como cualquier alteración que sufra el mismo, influirán en el estilo de vida de quienes se dedican a estas labores.

La ecología humana aborda la relación de los seres humanos con el ambiente. Para esta disciplina, el estudio de los estilos de vida de las comunidades es importante pues repercuten en diversos aspectos del uso de los recursos. En cuanto a la alimentación, por ejemplo, el enfoque ecológico se centra en el análisis del comportamiento alimentario y los requerimientos nutricionales según el contexto del ambiente físico y social de las poblaciones (Dufour y Teufel, 1995).

Los cambios ambientales afectan cada vez más la vida de las comunidades de campesinos. El cambio climático, altera la producción de cultivos al variar radicalmente los regímenes de temperaturas y lluvias, comprometiendo así la seguridad alimentaria tanto a nivel local como mundial (Easterling et al., 2007). Un elemento que hace distintiva a la pesca, es que los pescadores tienen poco control sobre la reproducción de los recursos marinos, desarrollan su actividad en un medio aleatorio, en el que el trabajo depende de los conocimientos y la experiencia, las condiciones ambientales, las características de las especies (movilidad y ciclo reproductivo) y la economía (Quezada, 2009). Las comunidades de pescadores son también susceptibles a las condiciones ambientales que afectan directamente sus ingresos. Es necesario destacar que muchas de estas poblaciones están activamente respondiendo a las condiciones climáticas y han demostrado innovación y resiliencia frente al cambio climático.

En la alimentación influye el desarrollo socioeconómico y en particular, los productos aportados por el medio concreto en que se asienta una comunidad dada. Históricamente las publicaciones sobre el uso de los recursos para la subsistencia de las poblaciones locales, y en particular de los pescadores y campesinos, provienen de diferentes áreas del conocimiento como la sociología y la geografía humana. Estos estudios están direccionados fundamentalmente a actividades específicas como la pesca y la agricultura. Dichas pesquisas pueden brindar información en dos sentidos: primero, en cuanto al manejo local de recursos utilizados para la subsistencia, y segundo, en cuanto a la calidad nutricional de la dieta de las poblaciones locales. La escasez de investigaciones con este enfoque, y con la perspectiva de la ecología humana en general, refleja la necesidad de concentrar los esfuerzos en abordajes de la integración de factores biológicos y ecológicos con los sociales (Hanazaki y Begossi, 2004).

El objetivo del presente trabajo es comparar variables del estilo de vida de un grupo de campesinos y pescadores ocasionales, del municipio Yaguajay, en la provincia de Sancti Spíritus, específicamente en relación con su dieta, estado nutricional y práctica de actividad física.

\section{MATERIAL Y MÉTODOS}

El municipio Yaguajay se encuentra al norte de la provincia de Sancti Spíritus, en el centro de Cuba. El grupo de campesinos fue seleccionado de la comunidad rural La Picadora; que se localiza a $22 \mathrm{~km}$ de la cabecera municipal, coordenadas $22^{\circ} 14$ ' 12 " N y $79^{\circ} 5^{\prime} 52^{\prime}$ ' W. La fuente de ingresos más importante de los habitantes se relaciona con las producciones agropecuarias. En el área hay 227 habitantes, de estos, 60 son hombres adultos entre 20 y 59 años, de ellos, 22 son campesinos y fueron escogidos 19 con el criterio de voluntariedad para participar en el estudio.

A su vez, en Yaguajay hay 47 personas inscritas en la Federación de pesca deportiva, de ellas 36 tienen entre 20 y 59 años. De las 27 personas convocadas, accedieron a participar en la investigación 14 hombres adultos y 2 mujeres, todos de procedencia urbana. Solo uno tiene 60 años y fue incluido en la muestra. La información de las dos mujeres fue usada exclusivamente en los temas relacionados con la alimentación, no en la evaluación de la actividad física y el estado nutricional, por el reducido tamaño de muestra.

Los pescadores que participaron son ocasionales, es decir no se dedican profesionalmente a la pesca, sino que realizan esta labor principalmente los fines de semana, y cuentan con un permiso de guardafronteras de un máximo de 36 
horas de estancia en el mar. En la encuesta se indagó sobre cuáles eran las otras actividades laborales que realizaban tanto pescadores como campesinos. El tipo de pesca que hacen es artesanal (Alcalá, 1999), basada fundamentalmente en la extracción de los recursos en zonas costeras y aguas interiores, como esteros, lagunas y ríos, mediante artes de pesca manuales, y en embarcaciones pequeñas, que pueden o no tener motor integrado.

El estudio realizado es de tipo descriptivo y para comparar la composición de la dieta de pescadores y campesinos se aplicó un cuestionario, empleado por el entonces Instituto de Nutrición e Higiene de los Alimentos de Cuba (INHA). Todos los participantes dieron su consentimiento informado y se cumplieron los principios éticos y las directrices de la Declaración de Helsinki sobre la protección de los humanos en la investigación.

El cuestionario tiene en cuenta el consumo semanal de los siete grupos básicos que se consideran en Cuba: Grupo I (cereales y viandas); Grupo II (vegetales); Grupo III (frutas); Grupo IV (carnes, aves, pescados, huevos y frijoles); Grupo V (lácteos); Grupo VI (grasas u otros alimentos fuentes de grasas); Grupo VII (azúcares). Es válido aclarar que el termino viandas se usa en Cuba para los tubérculos, raíces feculentas y frutas como plátanos y calabaza.

La composición de las dietas se determinó a partir de los 20 alimentos evaluados dentro de los 7 grupos: arroz, panes y galletas, pastas y viandas (Grupo I); vegetales (Grupo II); frutas (Grupo III); cerdo, pollo, embutidos, pescado, vísceras, huevos y granos (Grupo IV); leche, yogurt y quesos (Grupo V); grasa vegetal y grasa animal (Grupo VI); dulces y refrescos (Grupo VII).

Se analizó la frecuencia de su consumo desde 1-6 (1, todos los días; $2,4-5$ veces; $3,2-3$ veces; 4, 1 vez; 5, rara vez; 6 , nunca). Los 20 elementos fueron reducidos utilizando un Análisis Factorial con rotación varimax. Este análisis es capaz de detectar el comportamiento similar de múltiples variables y separarlas del resto. En nuestro caso, como se trata de frecuencia de consumo de alimentos, los factores van a detectar aquellos alimentos que son consumidos con determinada frecuencia. De esta forma, se determinan los diferentes componentes de una dieta. Para evaluar la consistencia de los factores se utilizó el índice Kaiser-Meyer-Olkin KMO, que permite evaluar la relevancia de cada variable en el análisis. Los criterios son: $\mathrm{KMO} \geq 0,75 \Rightarrow$ bien, $\mathrm{KMO} \geq 0,5 \Rightarrow$ aceptable y $\mathrm{KMO}<0,5 \Rightarrow$ inaceptable. Se desarrollaron análisis de U de Mann Whitney y t de Student para comparar las contribuciones medias de los dos grupos (campesinos y pescadores) a cada factor, es decir, dieta.

Las mediciones antropométricas fueron realizadas en octubre de 2017 a los 19 campesinos y a los 14 pescadores seleccionados e incluyeron peso, estatura, pliegues en hemicuerpo derecho del tríceps, bíceps, subescapular y suprailíaco, según las técnicas de la Convención Antropométrica de Airlie (Lohman, Roche y Martorell, 1988). Los instrumentos empleados fueron la balanza digital SECA para el peso corporal, el estadiómetro PROMES portátil para la estatura y de la marca Holtain la cinta métrica para las circunferencias y un calibrador de pliegues cutáneos. En el caso del pescador con 60 años y de un campesino con fractura de vértebra cervical, se estimó la estatura mediante las hemibrazas, con las ecuaciones para la población cubana (Díaz, 2011).

Se evaluó el estado nutricional por el índice de masa corporal (IMC), según los puntos de corte de la FAO (Shetty y James, 1994). Para estimar la composición corporal total, el cálculo de la densidad corporal se realizó con las ecuaciones para la suma de los pliegues cutáneos: bíceps, tríceps, subescapular y suprailíaco, según edad y sexo (Durnin y Womersley, 1974). El porcentaje de grasa se obtuvo con la ecuación de Siri (1961). Se utilizó la clasificación del porcentaje de grasa corporal total, teniendo en cuenta los puntos de corte de Bray, Davidson y Drenick (1972).

En septiembre de 2017 el municipio Yaguajay fue afectado por el huracán Irma, de categoría 5 en la escala de Saffir Simpson. Los pobladores aumentaron la actividad física debido a las labores de reconstrucción de las viviendas. En el caso de los campesinos, la actividad física se incrementó por otras razones: pérdida de cobertura boscosa que conllevó a la tala y recogida de árboles, cambios en las precipitaciones, que influyeron en las cosechas y en los ciclos de los cultivos, intensificación de las labores agrícolas, todo ello con alternancia de actividades de recuperación. Las afectaciones laborales de los pescadores fueron menores, pues la pesca se reanudó en función del estado del tiempo. Debido a este fenómeno, se realizaron en marzo del 
2018, las mismas mediciones antropométricas a una submuestra de 18 campesinos y 10 pescadores, que accedieron a participar en el monitoreo longitudinal de su estado nutricional, después de cinco meses de la evaluación inicial. El objetivo fue examinar el impacto de los cambios ecológicos en la región, sobre el estado nutricional de los pescadores y campesinos y el monitoreo culminó con esta segunda intervención.

Se calcularon las diferencias intragrupales entre los promedios finales e iniciales del peso, el índice de masa corporal y el porcentaje de grasa corporal. A esta submuestra también se le evaluó la actividad física global durante 7 días, mediante un cuestionario IPAQ versión corta y se indagó si ejercían otra ocupación. Los individuos fueron clasificados en sedentarios, irregularmente activos y activos (Ainsworth et al, 2000). Se determinaron los METS o equivalentes metabólicos. Estos representan la energía consumida mientras se permanece sentado en estado de reposo. Existen valores específicos de METS para diferentes actividades físicas, que se establecen en relación al reposo (caminar $=3,3$ METS, actividad física moderada=4 METS, actividad física vigorosa $=8$ METS, ciclismo modera$\mathrm{do}=7,5$ METS (Ainsworth et al, 2000, World Confederation for Physical Therapy, 2017)

El índice de actividad física se calculó como el producto de la intensidad en METS por la frecuencia por la duración y se expresa en METS*minuto a la semana y se clasificó en las categorías de alta, media y baja (Ainsworth et al., 2000).

Las variables discretas se analizaron con distribuciones de frecuencias y las continuas con la media y desviación estándar. El test de Kolmogorov - Smirnov se empleó para comprobar la normalidad de las variables y la prueba Levene para analizar la homogeneidad de varianzas. La comparación de los promedios de las variables continuas entre campesinos y pescadores se efectuó con la prueba t Student, con nivel de significación $p<0,05$, para muestras independientes en la evaluación inicial y para muestras pareadas en la comparación con la evaluación final. Los datos fueron procesados $\mathrm{y}$ analizados mediante el paquete estadístico SPSS versión 16.

\section{RESULTADOS}

De los 19 campesinos entrevistados, 4 desa- rrollaban otras labores además de la agricultura. Por otra parte, de los 14 pescadores, 13 tenían otras ocupaciones entre ellas: obrero, músico, albañil, chofer, maestro de educación física y marinero, siendo solo uno de ellos pescador a tiempo completo.

\section{Evaluación de la composición de las dietas}

El consumo de cereales es diario en el 100 $\%$ de los dos grupos, específicamente el arroz. Los frijoles son consumidos todos los días por el $89,5 \%$ de los campesinos y el $84,5 \%$ de los pescadores. El 58,0 \% de los campesinos y la mitad de los pescadores consumen diariamente viandas.

De los 20 alimentos evaluados se eliminaron aquellos que resultaron con valores menores de 0,5 en el análisis de Kaiser-Meyer-Olkin. Las variables restantes fueron sometidas al Análisis Factorial con rotación varimax y los alimentos con contribuciones inferiores a 0,4 también fueron descartados, quedando finalmente 11 alimentos (Tabla 1).

Los autovalores de los primeros tres factores detectados por el análisis estadístico explicaron el $60 \%$ de la varianza total, por ello se discriminaron tres tipos de dietas. Los valores numéricos en este análisis se distribuyen entre -1 y 1 . Mientras más se acerca el valor a 1 ó -1 , más importante y potente es la contribución del alimento a la dieta. El signo nos muestra si este alimento es consumido o no. Si el valor es positivo, el alimento es consumido y mientras más se acerque a 1 , se consume en mayor cantidad. Si el valor es negativo, se consume poco en esa dieta y mientas más se acerque a -1 menor es su consumo. De acuerdo a esta explicación, la primera dieta se caracterizó por elevado consumo de pescado, grasa vegetal y vegetales, pero bajo consumo de embutidos, granos y grasa animal; una segunda, con elevado consumo de vísceras y bajo de dulces, refrescos, panes y galletas y una tercera con altos niveles de consumo de panes y galletas y bajo consumo de carne de cerdo y granos.

$\mathrm{El}$ análisis de las contribuciones relativas de cada grupo en las dietas demostró que no existen diferencias significativas en las dietas dos y tres $(\mathrm{W}=180, \mathrm{p}=0,3625 \mathrm{y} \mathrm{t}=-0,16286, \mathrm{p}=0,8722$, respectivamente). Sin embargo, el consumo de la dieta uno mostró diferencias significativas, con 
TABLA 1. Resumen del análisis factorial de los componentes dietarios para pescadores y campesinos

\begin{tabular}{cccc}
\hline $\begin{array}{c}\text { Elementos } \\
\text { evaluados }\end{array}$ & Dieta 1 & Dieta 2 & Dieta 3 \\
\hline $\begin{array}{c}\text { Panes y galletas } \\
\text { Vegetales }\end{array}$ & 0,56 & $-0,4$ & 0,59 \\
$\quad$ Cerdo & & & $-0,82$ \\
Embutidos & $-0,59$ & & \\
Pescado & 0,74 & & \\
Vísceras & & 0,69 & \\
Grasa vegetal & 0,71 & & \\
Grasa animal & $-0,6$ & & \\
Dulces & & $-0,82$ & \\
Granos & $-0,51$ & & $-0,48$ \\
Refrescos & & $-0,84$ & \\
\hline Autovalores & 2,63 & 2,30 & 1,68 \\
$\%$ de varianza & 24 & 21 & 15 \\
\hline
\end{tabular}

una media de - 0,7077973 en el grupo de campesinos y 0,8405093 en el de pescadores $(t=-5,7079$; $\mathrm{p}<0,001$ ), como se aprecia en la Figura 1 del gráfico de cajas y bigotes para la primera dieta. De este modo, los pescadores mostraron alto consu- mo de pescado, vegetales y grasa de origen vegetal mientras que los campesinos consumieron más embutidos y grasa de origen animal.

\section{Comparación de variables e índices antropométricos a los cinco meses de la evaluación inicial en la submuestra de 18 campesinos y 10 pescadores}

A los cinco meses de la primera evaluación antropométrica se repitieron las mediciones y se aplicó el cuestionario IPAQ de actividad física a una submuestra de 18 campesinos y 10 pescadores que accedieron a continuar en la pesquisa. La disminución de la muestra es un hecho usual en los estudios longitudinales y en el presente trabajo se debió a que no todos los participantes iniciales asistieron el día de la medición.

En la Tabla 2 se expone la comparación de los promedios de las mediciones e índices antropométricos iniciales y finales en cada grupo. No existen diferencias significativas en las variables analizadas.

Con los resultados de la tabla 2 se calcularon las diferencias de los promedios finales e iniciales de las variables antropométricas e índices en cada grupo, las cuales aparecen reflejadas en la Figura 2. Se evidencia que los valores prome-

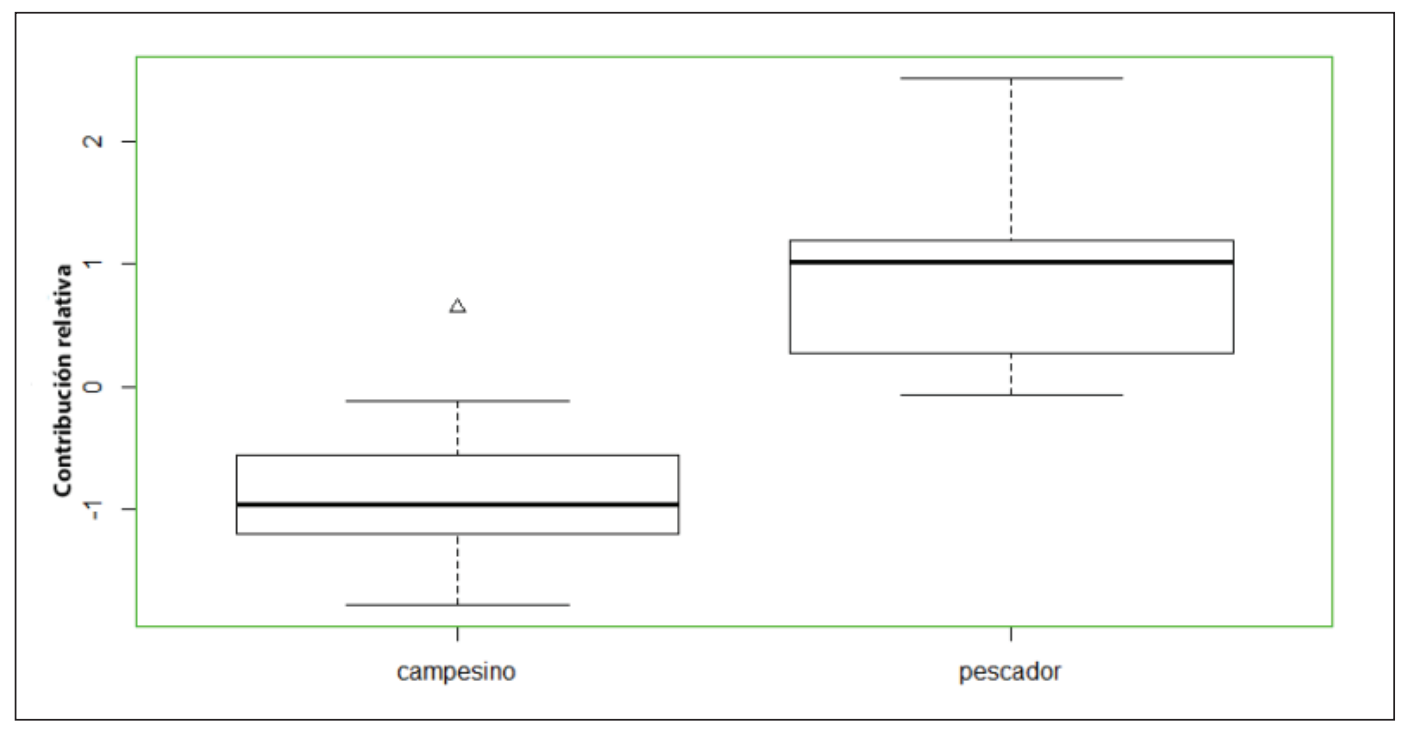

Fig. 1. Gráfico de cajas y bigotes de la contribución relativa de campesinos y pescadores a la dieta uno (medias: $-0,7077973$ en campesinos y 0,8405093 en pescadores). Las contribuciones relativas fueron calculadas utilizando un Análisis Factorial. 
$\boldsymbol{T A B L A}$ 2. Estadística descriptiva de las mediciones antropométricas e índices y comparación de los valores iniciales y finales en cada grupo

\begin{tabular}{|c|c|c|c|c|c|c|c|c|}
\hline & \multicolumn{4}{|c|}{ Campesinos } & \multicolumn{4}{|c|}{ Pescadores } \\
\hline & $\mathrm{X} \pm \mathrm{DE}$ & $\mathrm{X} \pm \mathrm{DE}$ & & & $\mathrm{X} \pm \mathrm{DE}$ & $\mathrm{X} \pm \mathrm{DE}$ & & \\
\hline Variable & $\begin{array}{l}\text { Evaluación } \\
\text { inicial }\end{array}$ & $\begin{array}{l}\text { Evaluación } \\
\text { final }\end{array}$ & $t$ Student & p, Sign. & $\begin{array}{l}\text { Evaluación } \\
\text { inicial }\end{array}$ & $\begin{array}{l}\text { Evaluación } \\
\text { final }\end{array}$ & t Student & p, Sign. \\
\hline & $\begin{array}{c}\text { Octubre } \\
2017\end{array}$ & Marzo 2018 & & & $\begin{array}{c}\text { Octubre } \\
2017\end{array}$ & Marzo 2018 & & \\
\hline Peso (kg) & $69,82 \pm 12,32$ & $68,91 \pm 11,14$ & 1.481 & $0,157 \mathrm{~ns}$ & $74,55 \pm 11,74$ & $74,64 \pm 11,82$ & -0.213 & $0,836 \mathrm{~ns}$ \\
\hline $\begin{array}{c}\mathrm{IMC}(\mathrm{kg} / \\
\left.\mathrm{m}^{2}\right)\end{array}$ & $23,50 \pm 3,83$ & $23,21 \pm 3,58$ & 1.49 & $0,155 \mathrm{~ns}$ & $25,51 \pm 3,82$ & $25,53 \pm 3,77$ & -0.136 & $0,895 \mathrm{~ns}$ \\
\hline $\begin{array}{c}\text { Porcentaje } \\
\text { de grasa } \\
\text { corporal }\end{array}$ & $20,33 \pm 7,12$ & $19,65 \pm 6,54$ & 1.399 & $0,18 \mathrm{~ns}$ & $23,62 \pm 6,09$ & $24,54 \pm 6,28$ & -1.548 & $0,156 \mathrm{~ns}$ \\
\hline
\end{tabular}

$\mathrm{X} \pm \mathrm{DE}:$ Media \pm desviación estándar $\mathrm{p}$, Sig : Nivel de significación de la prueba t Student ns: Diferencias no significativas

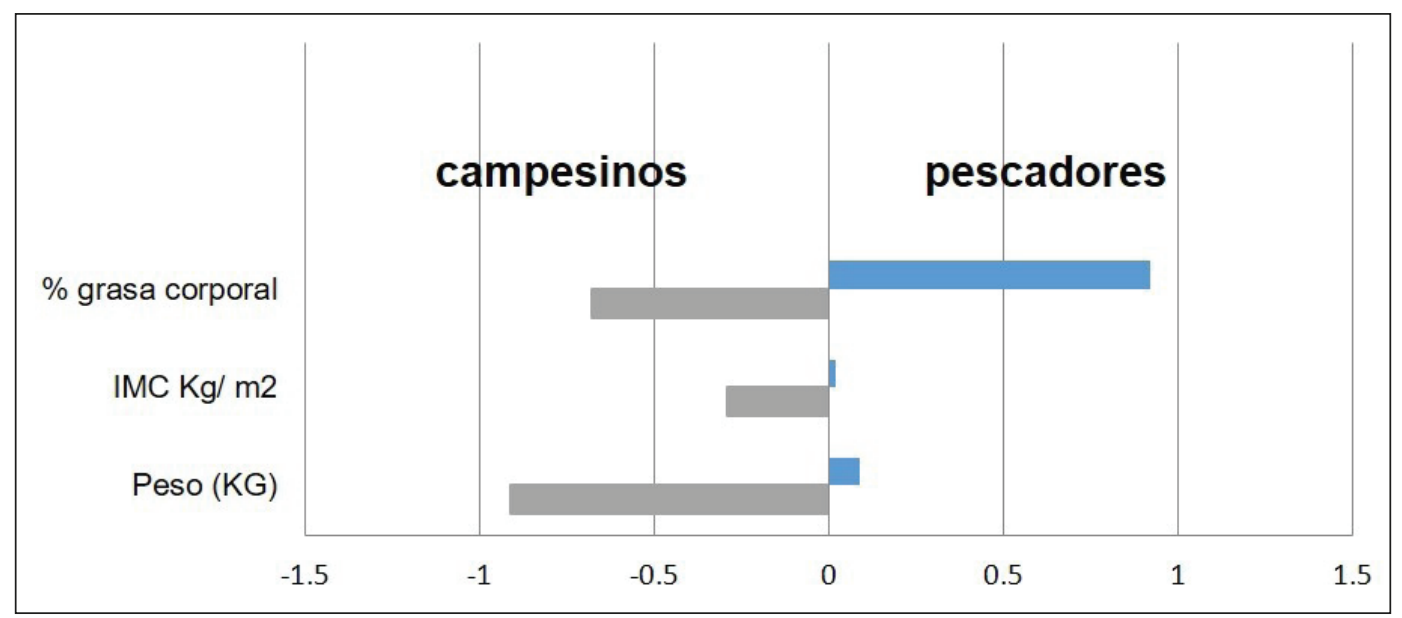

Fig. 2. Diferencias de los valores promedios finales e iniciales de las variables antropométricas e índices en cada grupo.

dios de los pescadores fueron mayores en la segunda medición respecto a la primera, situación contraria a lo ocurrido en los campesinos. En los primeros se incrementó en aproximadamente una unidad el porcentaje de grasa corporal, mientras que los segundos perdieron aproximadamente $1 \mathrm{~kg}$ de peso.

\section{Actividad física}

De acuerdo a la clasificación del cuestionario IPAQ, de actividad física durante 7 días, todos los pescadores y campesinos fueron clasificados como activos. En cuanto al índice de actividad física, los campesinos presentaron un valor promedio de 2919,79 \pm 14650 METS*minuto a la semana, mayor que el obtenido para los pescadores que fue de $18254,85 \pm 19824$ METS*minutos a la semana, sin diferencias significativas $(\mathrm{t}=1,73 \mathrm{y}$ $\mathrm{p}=0,095)$. El $60 \%$ de los pescadores fue clasificado con una categoría de actividad física alta y el $40 \%$ restante presentaba una actividad física media, las cifras para los campesinos fueron de $88,9 \%$ y $11,1 \%$ respectivamente. 


\section{DISCUSIÓN}

Todos los campesinos residen en zona rural, mientras que los pescadores son de zonas urbanas y habitan en la cabecera municipal de Yaguajay. Solo uno de estos últimos es pescador a tiempo completo, el resto tiene disímiles ocupaciones además de la pesca artesanal (chofer, profesor, labores administrativas, entre otras), en contraste con apenas 4 de los 19 campesinos, que se dedican a otras labores.

Los campesinos de la muestra están organizados en cooperativas de producción agropecuaria constituidas con las tierras y otros bienes aportados por los agricultores. Ellos cultivan, consumen e intercambian sus propios productos agropecuarios. $\mathrm{Al}$ igual que los pescadores y el resto de los cubanos, cuentan con una cartilla de racionamiento de alimentos básicos.

En ambos grupos analizados se consumen el arroz, los frijoles, las carnes y viandas como alimentos básicos, lo cual es característico de la comida tradicional de la zona rural del país (Núñez y González, 1995). Los pescadores, a pesar de ser urbanos, tienen un patrón alimentario caracterizado por la mezcla de elementos tradicionales de la comida rural cubana, combinado con el alto consumo de pescado, que no es característico de los campesinos. Por ello, es importante el análisis de las particularidades sociodemográficas y de los contextos ambientales donde viven las poblaciones, para describir su dieta. En la poca variedad de alimentos consumidos repercuten elementos como la dependencia del uso de recursos del medio, la influencia de la estacionalidad de los cultivos, el autoconsumo, la tradición cultural, el poco desarrollo de la industria conservera, la influencia del clima y la insuficiencia tecnológica.

En la literatura consultada no es frecuente el análisis del estado nutricional y la composición corporal de pescadores y campesinos, lo cual dificulta la comparación de los datos. Por ejemplo, en una pesquisa efectuada en tres comunidades rurales de la Reserva de la Biosfera Península de Guanahacabibes, en la provincia cubana de Pinar del Río (Rodríguez, 2007), se evaluó el estado nutricional de personas que realizaban labores de explotación, mantenimiento y conservación de los recursos naturales terrestres y marinos que ofrece el medio circundante. Las actividades implicaban trabajos fuertes como obreros, carboneros, pescadores, monteros, guarda parques, mantenimiento de viales, cortar leña, entre otras. Sin embargo, en este estudio no se realiza una distinción en cuanto a la ocupación de los habitantes, razón por la cual no se comparan los datos obtenidos, con los de pescadores y campesinos de Yaguajay.

En una investigación sobre nivel de sedentarismo en 41 pescadores artesanales de la región de Los Lagos, Chile, se obtuvo un IMC promedio de $30,84 \mathrm{~kg} / \mathrm{m}^{2}$ (Véliz, Dörner, y Ripoll, 2017) mayor a los reportados en el actual trabajo. Se debe tener en cuenta la limitante de que los pescadores encuestados de Yaguajay son ocasionales, es decir no se dedican profesionalmente a la pesca y la realizan mayormente los fines de semana, mientras que en el resto de los días se dedican a otros oficios.

Por otra parte, Pérez, Rosique, Turbay y Machado (2016) realizaron un análisis en 21 campesinos colombianos productores de café y la media de IMC fue de $22,1 \mathrm{~kg} / \mathrm{m}^{2}$, menor a las registradas en Yaguajay.

En una investigación realizada en zonas rurales de Australia, la muestra fue dividida de acuerdo a tres categorías ocupacionales (1 campesinos y pescadores, 2 técnicos y 3 , directivos) para analizar el riesgo de enfermedades cardiovasculares. Los valores obtenidos para el grupo 1 fueron superiores a los de la presente pesquisa pues el peso promedio fue de $87,6 \mathrm{~kg}$ y el IMC de $28,0 \mathrm{~kg} / \mathrm{m}^{2}$ (Davis-Lameloise et al., 2013).

La actividad física que realizan ambos grupos difieren. Los campesinos practican actividad física diariamente, efectuando labores en el campo como la siembra, el cultivo, la cosecha y el procesamiento de los productos agrícolas. Por su parte, los pescadores pescan ocasionalmente, generalmente los fines de semana y tienen ocupaciones disímiles que incluyen labores sedentarias. A pesar de que los resultados de las encuestas evidenciaron que el índice de actividad física fue superior para los campesinos, es necesario cuantificar esta variable con técnicas e instrumentos más precisos como los acelerómetros de los cuales el colectivo de autores no tiene disponibilidad.

A su vez, el paso del huracán Irma afectó los cultivos y el estado constructivo de las viviendas. Los campesinos tuvieron además que rea- 
lizar diferentes labores de recuperación agrícola y del paisaje. Estos factores pudieran explicar porque en la segunda medición perdieron como promedio $1 \mathrm{~kg}$ de peso al transcurrir seis meses del evento climatológico mientras que los pescadores incrementaron su porcentaje de grasa corporal en aproximadamente una unidad.

Se dificulta la comparación con estudios en otras poblaciones, debido a la escasa bibliografía sobre el tema, en especial en Cuba, a lo que se le suma el hecho de que los pescadores son ocasionales y no a tiempo completo. Otra limitación de la investigación es el reducido tamaño de la muestra. Se debe tener en cuenta que el total de habitantes de la comunidad rural La Picadora es de 227 habitantes y se midieron 19 de 22 posibles campesinos. Por otra parte, de los 27 posibles pescadores de Yaguajay convocados a participar en la pesquisa, solo asistieron a entrevistarse y a realizarse las mediciones 14 individuos.

\section{CONCLUSIONES}

El estilo de vida de los dos grupos analizados se caracterizó por dietas con alto consumo de arroz, frijoles y viandas. Los campesinos practican actividad física intensa diariamente en la agricultura y tienen menor obesidad, mientras que los pescadores pescan ocasionalmente. A su vez, estos últimos tienen una dieta más saludable, con mayor consumo de pescado y vegetales.

\section{LITERATURA CITADA}

Ainsworth, A., Haskell, W., Whitt, M., Irwin, M., Swartz, A., Strath, S., O’Brien, D y Leon, A. (2000). Compendium of physical activities: an update of activity codes and METs intensities, Med Sci Sports Exercise, 32, 498516. doi:10.1097/00005768-200009001-00009

Alcalá, G. (1999). Con el agua hasta los aparejos: pescadores y pesquerías en el Soconusco, Chiapas, México.

Álvarez, L. (2009). Estilos de vida alimentación. Gaceta Antropología, 25, 25-27.

Bray, G. A., Davidson, M. B y Drenick, E. J. (1972). Obesity: A serious symptom. UCLA Conference. Ann. Inter. Med. 77, 797-805. doi:10.7326/0003-4819-77-5-779

Davis-Lameloise, N., Philpot, B., Janus, E. D., Versac, E. D., Laatikainen, T., Vartiainen, E.A y Dunbar, J. (2013).
Occupational differences, cardiovascular risk factors and lifestyle habits in South Eastern rural Australia. BMC Public Health, 13, 1-7. doi:10.1186/1471-245813-1090

Díaz, M.E. (2011). Indicadores antropométricos de la evaluación nutricional. En Alimentación, nutrición y salud. La Habana: Cámara del Libro.

Dufour, D.L y Teufel, N.I. (1995). Minimum data sets for the description of diet and measurement of food intake and nutritional status. En The comparative analysis of Human Societies: Toward Common Standards for Data Collection and Reporting, Lynne Rienner Boulder.

Durnin, J.V y Womersley, J. (1974). Body fat assessed from total body density and its estimation from skinfold thickness measurements on 481 men and women aging from 16 to 72 years. Brit. J. Nutr, 32, 77-97. doi:10.1079/BJN19740060

Easterling, W.E., Aggarwal, P.K., Batima, P., Brander, K.M., Erda, L., Howden, S. y Tubiello, F.N. (2007). Food, fibre and forest products. En Climate Change: Impacts, Adaptation and Vulnerability, pp. 273-313. Cambridge, Reino Unido. Eds. Cambridge University Press.

Hanazaki, N y Begossi, A. (2004). Dieta de populações de pescadores. En Begossi, A. (Organizadora).Ecología de Pescadores da Mata Atlâantica e da Amazonia. Brasil, (pp. 149-166).

Instituto de Nutrición e Higiene de los Alimentos de Cuba. INHA. (2004) Guías alimentarias para la población cubana mayor de dos años de edad. Biblioteca Virtual del Instituto de Nutrición. Recueperado de: http:/www. inha.sld.cu/bvs/guias_alimentarias/guia1

Lohman, T.G., Roche, A.F yMartorell, R. (1988). Anthropometric Standardization Reference Manual. Human Kinetics Books Champaign, Illinois:.USA. .

Núñez, N y González, E. (1995). Diferencias regionales en las comidas tradicionales de la población rural de Cuba. Rev. Cubana. Aliment. Nutr, 9 (2), 79-93.

Pérez, F., Rosique, J., Turbay, S y Machado, M. (2016). Estudio de la seguridad alimentaria y nutricional de unidades campesinos productoras de café en rediseño agroecológico (Cuenca del río Porce, Antioquía). Agroalimentaria, 22 (42), 171-189.

Quezada, F. (2009). Hacia una Antropología de las sociedades costeras. Catauro Revista cubana de antropología, 19, 17-28

Rodríguez, D. (2007). Ecología humana en la Reserva de la Biosfera Península de Guanahacabibes. Estudio transversal de tres comunidades. Tesis de Maestría en Antropología. Universidad de La Habana

Shetty, P.S y James, W.P. (1994). Body mass index: A measure of chronic energy deficiency in adults, FAO. Food and Nutrition Papers.

Siri, W.E. (1961). Apparatus for measuring human body volum. Rev. Sci. Instruments, 27, 279-238.

Véliz, A., Dörner, A y Ripoll, M. (2017). Bienestar sicológico y nivel de sedentarismo de pescadores artesanales de la Región de Los Lagos, Chile. Humanidades Médicas, $17(2), 323-337$.

World Confederation for Physical Therapy, 2017. Recuperado de:https://www.wcpt.org/congress 